\title{
Engineering edge-state currents at the interface between narrow ribbons of two-dimensional topological insulators
}

\author{
H. Ishida $\odot^{1}$ and A. Liebsch ${ }^{2}$ \\ ${ }^{1}$ College of Humanities and Sciences, Nihon University, Tokyo 156-8550, Japan \\ ${ }^{2}$ Peter Grünberg Institut and Institute for Advanced Simulations, Forschungszentrum Jülich, 52425 Jülich, Germany
}

(Received 8 April 2020; accepted 15 May 2020; published 28 May 2020)

\begin{abstract}
We consider a junction between two semi-infinite narrow ribbons of a two-dimensional (2D) topological insulator (TI) and calculate the electron current through the interface carried by one-dimensional (1D) edge states formed in the bulk band gap. In addition to an abrupt interface between ribbons, we also consider a configuration where one or two rectangular islands of finite length are sandwiched between these ribbons. We demonstrate that it is feasible to create "current-on" and "current-off" states by modifying the energy dispersion of $1 \mathrm{D}$ interface edge states and the resonant levels localized at the boundary of the island regions.
\end{abstract}

DOI: 10.1103/PhysRevResearch.2.023242

\section{INTRODUCTION}

Since the epoch-making proposal for the quantum spin Hall (QSH) phase in graphene with nonzero $Z_{2}$ topological invariant by Kane and Mele [1,2], the research on new classes of materials with nontrivial topological orders in momentum space has made rapid progress [3-8]. The QSH insulator, termed also the two-dimensional (2D) topological insulator (TI), was first realized in $\mathrm{CdTe} / \mathrm{HgTe} / \mathrm{CdTe}$ quantum well heterostructures [9-11]. Subsequent calculations [12] and experiments have revealed that various $2 \mathrm{D}$ materials such as bismuth bilayer [13-16], stanene [17-19], and transitionmetal dichalcogenide monolayers [20-23] exhibit the QSH phase.

From an application viewpoint, an important feature of 2D TIs is the existence of helically spin-polarized onedimensional (1D) edge states that propagate unidirectionally along the boundary of the 2D crystal. In momentum space, they form a Kramers pair of 1D metallic bands crossing the bulk band gap whose energy dispersion curves intersect each other at a time-reversal invariant momentum point in the 1D Brillouin zone. Since the backscattering of electrons from one branch to the other of the two bands is forbidden due to the orthogonality of two states forming a Kramers pair, the QSH edge states provide dissipationless 1D conduction channels that are robust against nonmagnetic defects [2,24-29].

In the past, several proposals were made for topological transistors that made use of QSH edge channels [20,30-32]. Typically, the current in these devices is controlled by applying an electric or magnetic field that induces phase transitions, through which a finite energy gap is opened in the edge

Published by the American Physical Society under the terms of the Creative Commons Attribution 4.0 International license. Further distribution of this work must maintain attribution to the author(s) and the published article's title, journal citation, and DOI. channels, thus leading to current-off states. Among other mechanisms, Xu et al. [33] proposed a topological transistor in which tunneling probabilities between the $1 \mathrm{D}$ edge channel forming part of the source region and another one, oriented parallel to the former and forming part of the drain region, are tuned between 0 and 1 through a transverse electric field within the $2 \mathrm{D}$ plane.

In the present work, we consider nanojunctions made out of narrow ribbons of 2D TIs. In the simplest case, the system consists of two semi-infinite narrow ribbons of 2D TIs that are coupled weakly at their ends. By modifying the coupling strength and topological invariants of both ribbons, one can open a gap or change the energy dispersion of 1D edge channels at the interface, which results in large variations of tunneling conductance. Furthermore, we consider nanojunctions in which one or more rectangular islands of 2D TIs of finite size are sandwiched between the two semi-infinite ribbons and weakly coupled to them. The 1D edge channels in the rectangular islands are discretized due to finite size effects and give rise to sharp resonant structures in the tunneling conductance across the junction. Based on numerical results, we discuss possible applications of the present 2D TI junctions for topological transistors.

The outline of this paper is as follows. In the next section, we present the theoretical model and the method for evaluating the tunneling current through the nanojunction. Section III contains the discussion of the results. A conclusion is given in Sec. IV.

\section{MODEL AND METHOD}

To represent 2D TIs, we adopt a two-band tight-binding Hamiltonian on a square lattice known as the BernevigHughes-Zhang (BHZ) model [9]. The two sides of the square unit cell are chosen as the $x$ and $y$ axes, and the $z$ axis points normal to the 2D plane. For simplicity, we employ the original BHZ Hamiltonian, which is diagonal with respect to the $z$ component of electron spin, although it can be generalized to 

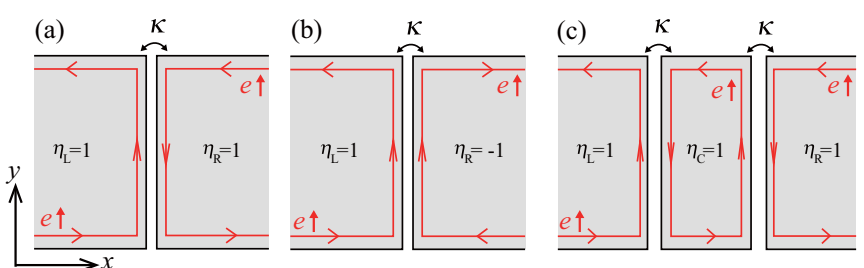

FIG. 1. Nanojunctions considered in the present work. In panels (a) and (b), two semi-infinite ribbons of the 2D TI modeled by the BHZ Hamiltonian are connected at their ends. In panel (c), a rectangular ribbon of the $2 \mathrm{D} \mathrm{TI}$ is sandwiched between the two semiinfinite ribbons. Electron hopping integrals across the neighboring ribbons are scaled with respect to bulk values by parameter $\kappa . \eta_{L}, \eta_{R}$, and $\eta_{C}$ are Chern numbers of the valence band for up-spin electrons in each ribbon. Solid lines and red arrows indicate up-spin edge channels when $\kappa=0$.

spin nondiagonal Hamiltonians by adding symmetry-breaking terms [34,35].

The Hamiltonian is specified by the on-site energies of $s$ and $p$ orbitals, $\varepsilon_{s}$ and $\varepsilon_{p}$, and the nearest-neighbor (NN) hopping integral for $\sigma$-bonding between two $s$ orbitals, $t_{s s}$, between two $p$ orbitals, $t_{p p}$, and between $s$ and $p$ orbitals, $t_{s p}$. The $2 \times 2$ Hamiltonian for up-spin electrons is then constructed by choosing the $s$ and $\left(p_{x}+i p_{y} \eta\right) / \sqrt{2}$ orbitals on each lattice site as basis functions with the parameter $\eta= \pm 1$. In real space, the on-site Hamiltonian matrix for site $\mathbf{x}=(x, y)$ is

$$
\hat{\mathcal{H}}_{\mathbf{x}, \mathbf{x}}=\left(\begin{array}{cc}
\varepsilon_{s} & 0 \\
0 & \varepsilon_{p}
\end{array}\right),
$$

while the hopping Hamiltonian matrix between two NN sites, $\mathbf{x}$ and $\mathbf{x}^{\prime}$, is given by

$$
\hat{\mathcal{H}}_{\mathbf{x}, \mathbf{x}^{\prime}}=\left(\begin{array}{cc}
t_{s s} & -e^{i \theta \eta} t_{s p} \\
e^{-i \theta \eta} t_{s p} & t_{p p}
\end{array}\right),
$$

where $\theta$ specifies the angle of rotation of vector $\mathbf{x}^{\prime}-\mathbf{x}$ relative to the positive $x$ direction. In momentum space $\mathbf{k}=\left(k_{x}, k_{y}\right)$, the Hamiltonian for a bulk crystal is given by

$\hat{\mathcal{H}}(\mathbf{k})$

$$
=\left(\begin{array}{cc}
\varepsilon_{s}+2 t_{s s}\left(\cos k_{x}+\cos k_{y}\right) & 2 t_{s p}\left(-i \sin k_{x}+\eta \sin k_{y}\right) \\
2 t_{s p}\left(i \sin k_{x}+\eta \sin k_{y}\right) & \varepsilon_{p}+2 t_{p p}\left(\cos k_{x}+\cos k_{y}\right)
\end{array}\right),
$$

where the lattice constant is chosen to be the unit length. As is well known, this Hamiltonian represents a 2D TI when the band inversion condition $\varepsilon_{p}-\varepsilon_{s}<4\left(\left|t_{s s}\right|+\left|t_{p p}\right|\right)$ is fulfilled [36,37]. In this case the sign of $\eta$ determines the Chern number of the valence band. The Hamiltonian for down-spin electrons with the opposite Chern number is obtained by reversing the sign of $\eta$ in Eq. (2).

In the present work we investigate electron tunneling at the interface between two semi-infinite ribbons of 2D TIs represented by the BHZ model. Figure 1 depicts the three interface structures we consider. The left and right ribbons are semi-infinite in the $x$ direction and comprise $N_{y}$ lattice points in the $y$ direction. All Hamiltonian parameters are assumed to be the same as the ones in the bulk except for those at the interface. Figures 1(a) and 1(b) show abrupt interfaces in which the NN hopping matrix $\hat{\mathcal{H}}_{\mathbf{x}, \mathbf{x}^{\prime}}$ in Eq. (2) is replaced by $\kappa \hat{\mathcal{H}}_{\mathbf{x}, \mathbf{x}^{\prime}}$ by multiplying by a factor $\kappa(0 \leqslant \kappa \leqslant 1)$ for electrons tunneling across the two ribbons. Experimentally, one could insert a thin layer of a large band-gap trivial insulator between the two ribbons to weaken the coupling between them. The Chern number of the valence band in the left (right) ribbon is denoted by $\eta_{L}\left(\eta_{R}\right)$, which determines whether the edgestate electrons propagate along the boundary anticlockwise or clockwise as seen from the positive $z$ side. There are two possible cases with $\eta_{L}=\eta_{R}$ in Fig. 1(a) and $\eta_{L}=-\eta_{R}$ in Fig. 1(b).

Figure 1(c) shows a geometry in which a rectangular island consisting of $N_{x} \times N_{y}$ lattice points is sandwiched between the left and right ribbons, where $\kappa$ is the scaling parameter of the $\mathrm{NN}$ hopping integrals between neighboring ribbons, and $\eta_{C}$ determines the Chern number of the island region. When $\kappa=$ 0 , the edge channel in the island consists of discrete states whose energy levels can be varied by changing $N_{x}$ and $N_{y}$. For $\kappa>0$, these discrete states become resonant levels that contribute to the resonant tunneling of electrons through the junction.

We also consider interface geometries in which two island regions with different $N_{x}$ values are inserted between the left and the right semi-infinite ribbons.

In all cases, down-spin edge states that propagate in the direction opposite that of the up-spin ones are not depicted in Fig. 1. Since the Hamiltonian in the present work is spin diagonal, up-spin and down-spin electrons make the same contribution to the electron conductance. Hereafter, we consider only up-spin electrons.

Let us consider an edge-state electron with energy $\varepsilon$, which is incident from the interior of the left ribbon, elastically scattered at the interface, and partly transmitted to the interior of the right ribbon. For example, in Fig. 1(a), the incident electron wave that propagates along the lower edge of the left ribbon toward the right is scattered at the interface. Then, the backscattered electron wave propagates along the upper edge of the left ribbon toward the left, whereas the transmitted electron wave propagates along the lower edge of the right ribbon toward the right. In contrast, in Fig. 1(b), the transmitted electron wave propagates along the upper edge of the right ribbon.

To calculate the electron transmittance, we employ the embedded Green's function technique [38]. In this method, one treats only a finite number of "layers" (1D chain consisting of $N_{y}$ sites in the present model) in the interface region explicitly, while the effects of the semi-infinite leads on both sides are expressed in terms of complex embedding potentials (termed more often lead self-energies $[39,40]), \hat{\Sigma}_{L}(\varepsilon)$ and $\hat{\Sigma}_{R}(\varepsilon)$, which are calculated from the wave functions of generalized Bloch states that propagate or decay toward the interior of the semi-infinite lead [41]. The Green's function in the interface region is given by

$$
\hat{G}(\varepsilon)=\left[\varepsilon \hat{I}-\hat{\mathcal{H}}-\hat{\Sigma}_{L}(\varepsilon)-\hat{\Sigma}_{R}(\varepsilon)\right]^{-1},
$$

where $\hat{\mathcal{H}}$ denotes the $\left(2 M_{x} N_{y}\right) \times\left(2 M_{x} N_{y}\right)$ tight-binding Hamiltonian for the embedded slab region with $M_{x}$ being the number of embedded layers. With use of $\hat{G}$ and $\hat{\Sigma}_{L(R)}$, the transmittance times the number of conduction channels for 

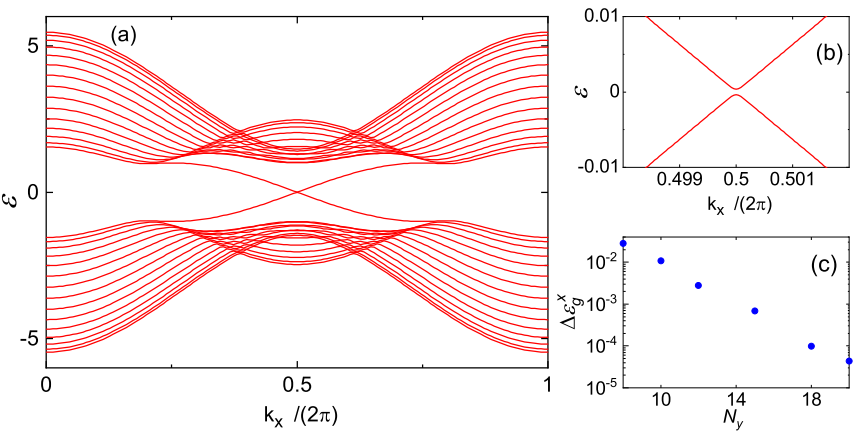

FIG. 2. (a) Energy levels versus wave number $k_{x}$ of a nanoribbon which is periodic in the $x$ direction and of finite width $N_{y}=15$ represented by the BHZ Hamiltonian with parameters $\varepsilon_{s}=-1.5$, $\varepsilon_{p}=1.5, t_{s s}=-1, t_{p p}=1$, and $t_{s p}=0.5$. The two bands crossing the bulk band gap are edge states localized at either the lower edge or the upper edge of the ribbon. (b) Magnification of panel (a) in the neighborhood of $k_{x}=\pi$ revealing a small energy gap between the two edge channels. (c) $\Delta \varepsilon_{g}^{x}$, the energy gap between the two edge modes at $k_{x}=\pi$, as a function of the ribbon width $N_{y}$.

electrons incident from the interior of the left ribbon with energy $\varepsilon$ is given by $[39,40]$

$$
T(\varepsilon)=\operatorname{Tr}\left[\hat{\Gamma}_{L}(\varepsilon) \hat{G}(\varepsilon) \hat{\Gamma}_{R}(\varepsilon) \hat{G}^{\dagger}(\varepsilon)\right]
$$

where $\hat{\Gamma}_{L(R)}=i\left(\hat{\Sigma}_{L(R)}-\hat{\Sigma}_{L(R)}^{\dagger}\right)$. Without scattering, $T(\varepsilon)$ is quantized to an integer $\mathcal{N}=0,1,2, \ldots(\mathcal{N}=1$ or 0 in the present model when $\varepsilon$ is within the bulk band gap). The ballistic current through the junction in the positive $x$ direction is given by

$$
I=\frac{2 e}{h} \int_{\mu_{R}}^{\mu_{L}} T(\varepsilon) d \varepsilon,
$$

where $\mu_{L}\left(\mu_{R}\right)$ is the chemical potential of the semi-infinite left (right) ribbon $\left(\mu_{L}>\mu_{R}\right)$ and the prefactor 2 accounts for the electron spin.

\section{RESULTS AND DISCUSSION}

Throughout the present work, we choose the Hamiltonian parameters as $\varepsilon_{s}=-1.5, \varepsilon_{p}=1.5, t_{s s}=-1, t_{p p}=1$, and $t_{s p}=0.5$. They satisfy the condition $\varepsilon_{p}-\varepsilon_{s}<4\left(\left|t_{s s}\right|+\left|t_{p p}\right|\right)$. In Fig. 2(a) we show the band structure of a 1D crystal of infinite length in the $x$ direction and finite width $N_{y}=15$ in the $y$ direction. Among the $2 N_{y}=30$ energy bands, the two bands crossing the bulk band gap are $1 \mathrm{D}$ edge states localized at the lower or upper side of the ribbon. Since the ribbon is of finite width, the two modes interact with each other due to the overlap of the exponential tails of wave functions, resulting in the opening of a small energy gap at the zone boundary $k_{x}=\pi$ as shown in Fig. 2(b). We plot in Fig. 2(c) the energy gap, $\Delta \varepsilon_{g}^{x}$, which is seen to decrease exponentially with increasing $N_{y}$. Since the electron transmittance $T(\varepsilon)$ is a symmetric function with respect to the band-gap center $\varepsilon=0$, we consider in the following edge-state electrons with $\varepsilon \geqslant \Delta \varepsilon_{g}^{x} / 2$.
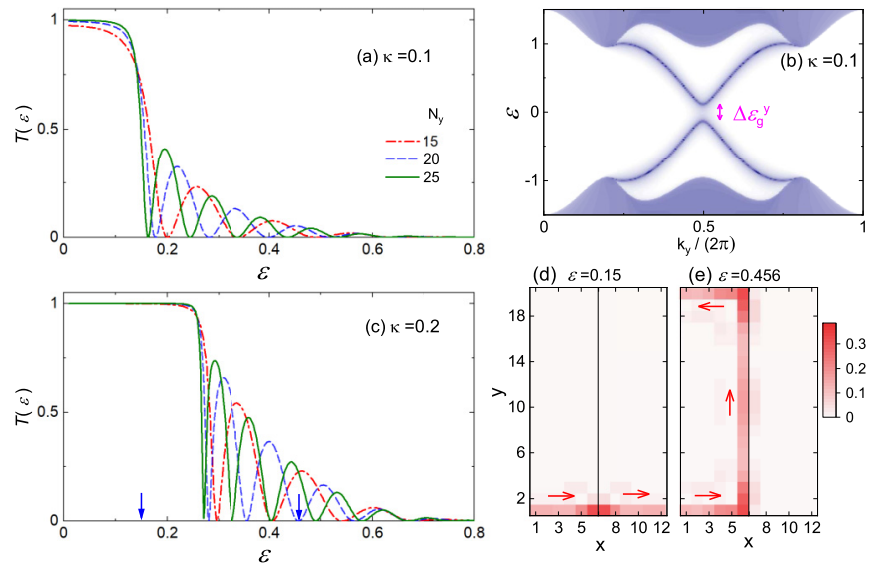

FIG. 3. (a) $T(\varepsilon)$ versus $\varepsilon$ of the nanojunction depicted in Fig. 1(a) for $\kappa=0.1$. Solid, dashed, and dot-dashed lines correspond to $N_{y}=25,20$, and 15 , respectively. (b) $\varepsilon$ versus wave number $k_{y}$ of interface edge states propagating in the $y$ direction along the interface for the junction in panel (a) in the limit of $N_{y} \rightarrow+\infty$. Lower and upper shaded regions are projected bulk valence and conduction bands, respectively. (c) The same as panel (a), except for $\kappa=0.2$. (d) Intensity map of $\sum_{i=s, p}|\psi(x, y, i)|^{2}$, the charge density of the 1D edge state incident from $x=-\infty$ with $\varepsilon=0.15$ marked by the left vertical arrow in panel (c). $N_{y}=20$ and $\kappa=0.2$. Red arrows indicate wave propagation directions. (e) The same as panel (d), except for $\varepsilon=0.456$ marked by the right vertical arrow in panel (c).

\section{A. Junctions between two semi-infinite ribbons with the same $\eta$ value}

We begin with the configuration shown in Fig. 1(a) where two semi-infinite 2D TI ribbons with the same topological invariant are coupled at their ends. For the coupling strength $\kappa=1$, the system becomes an infinite 1D crystal with no defects so that $T(\varepsilon)=1$ for any value of $\varepsilon$, while for $\kappa=0$, the system becomes two uncoupled ribbons so that $T(\varepsilon)=0$. We investigate how $T(\varepsilon)$ behaves when $\kappa$ lies between these two values.

In Fig. 3(a) we show $T(\varepsilon)$ as a function of $\varepsilon$ for three $N_{y}$ values. Although the coupling strength in this figure, $\kappa=0.1$, is rather small, we observe a transmittance plateau with $T(\varepsilon) \simeq 1$ on the low-energy side. This behavior can be explained by the energy dispersion of the edge modes that propagate in the $y$ direction along the interface. We show in Fig. 3(b) their dispersion curves for $\kappa=0.1$ in the wide ribbon limit $\left(N_{y} \rightarrow+\infty\right)$. When $\kappa=0$, the edge state of the left ribbon propagates in the positive $y$ direction, while that of the right ribbon propagates in the negative $y$ direction as illustrated in Fig. 1(a). For nonzero $\kappa$ values, these two channels are hybridized with each other, leading to the opening of an energy gap with size $\Delta \varepsilon_{g}^{y}$ as seen in Fig. 3(b). Within the energy range $|\varepsilon|<\Delta \varepsilon_{g}^{y} / 2$, no edge states propagating in the $y$ direction exist. Thus, electrons incident from $x=-\infty$ along the lower edge of the ribbon are perfectly transmitted across the junction, leading to $T(\varepsilon)=1$ when $N_{y}=+\infty$. For finite $N_{y}$ values, the two edge channels at the lower and upper boundaries of the left ribbon can still be coupled via the evanescent edge mode that decays toward the positive $y$ direction. As a result, incident electrons can partially be 
reflected toward $x=-\infty$ through the upper edge channel of the ribbon. This is the reason why $T(\varepsilon)$ within the interval $\varepsilon<\Delta \varepsilon_{g}^{y} / 2$ becomes smaller than unity with decreasing $N_{y}$. Since $\Delta \varepsilon_{g}^{y}$ increases almost linearly with increasing $\kappa$, the transmittance plateau with $T(\varepsilon) \simeq 1$ widens with increasing $\kappa$. This is seen in Fig. 3(c) where $T(\varepsilon)$ is plotted for $\kappa=0.2$.

In order to visualize the behavior of electron wave functions, we plot in Figs. 3(d) an intensity map of the squared amplitude of the electron wave function summed over two orbitals, $\sum_{i=s, p}|\psi(x, y, i)|^{2}$, on the $x y$ plane for $N_{y}=20$ at $\varepsilon=0.15$ marked by a vertical arrow in Fig. 3(c). It is seen that the electron incident from the left ribbon is perfectly transmitted to the right ribbon.

For $\varepsilon>\Delta \varepsilon_{g}^{y} / 2$, the incident electron wave can either propagate toward the positive $y$ direction through the interface edge channel or tunnel to further propagate toward $x=+\infty$ through the edge channel at the lower boundary of the right ribbon. It is seen from Figs. 3(a) and 3(c) that $T(\varepsilon)$ decays with increasing $\varepsilon$, exhibiting at the same time oscillating behavior with several zeros at which electrons are perfectly reflected. These are denoted by $\varepsilon_{1}, \varepsilon_{2}, \varepsilon_{3}, \ldots$ in ascending order.

As an example, we plot in Fig. 3(e) the squared amplitude of the electron wave function at $\varepsilon_{3}=0.456$ for $N_{y}=20$ marked by a vertical arrow in Fig. 3(c). It is seen that the charge density is distributed symmetrically with respect to $y=\left(N_{y}+1\right) / 2$. This also holds for the other zeros of $T(\varepsilon)$. The reason for this behavior is as follows. Let us denote the up-spin electron wave function incident from $x=-\infty$ along the lower edge of the left ribbon at $\varepsilon=\varepsilon_{n}$ by $\psi(x, y, i)$. By applying the time-reversal operator $\hat{T}$ on $\psi$, we obtain a downspin electron wave function, $\hat{T} \psi=\psi^{*}(x, y, i)$, which is incident from $x=-\infty$ along the upper edge of the left ribbon and perfectly reflected at the interface. On the other hand, because of the present geometrical symmetry, by applying $\hat{R}_{y}$, mirror reflection with respect to $y=\left(N_{y}+1\right) / 2$ on $\psi$, we obtain the same down-spin electron wave function, $\hat{R}_{y} \psi=\psi\left(x, N_{y}+\right.$ $1-y, i)$. Thus, we have $\psi\left(x, N_{y}+1-y, i\right)=c \psi^{*}(x, y, i)$ with a constant $c(|c|=1)$. Hence, at zeros of $T(\varepsilon)$, the charge density becomes symmetric with respect to $y=\left(N_{y}+1\right) / 2$, i.e., $\sum_{i=s, p}\left|\psi_{i}(x, y)\right|^{2}=\sum_{i=s, p}\left|\psi_{i}\left(x, N_{y}+1-y\right)\right|^{2}$.

The charge density in Fig. 3(e) exhibits three maxima along the interface. More generally, we have found that the charge density at $\varepsilon=\varepsilon_{n}$ exhibits $n$ maxima distributed with nearly the same interval along the $N_{y}$ wide interface in the $y$ direction. Except near the lower and upper corners of the left ribbon, $\psi(x, y, i)$ along the boundary line of the two ribbons may be expressed by a linear combination of two interface edge states with wave numbers $k_{y}=\pi \pm \Delta k_{n}$, which are given as the intersections of the energy dispersion curve of the upper interface band shown in Fig. 3(b) and $\varepsilon=\varepsilon_{n}$. Then, the interval between two maxima of $|\psi(x, y, i)|^{2}$ is given by $\pi / \Delta k_{n}$. As stated above, this interval is approximately equal to $N_{y} /(n-1)$. Hence, we have $\Delta k_{n} \simeq$ $\pi(n-1) / N_{y}$. Since the dispersion curve of the upper interface band in Fig. 3(b) is, roughly speaking, a linear function of $k_{y}$ except for its minimum at $k_{y}=\pi$, this also implies that $\varepsilon_{n}$ appears approximately with the same energy interval and further that this interval decreases inversely proportional to $N_{y}$. Both features are indeed seen in Figs. 3(a) and $3(\mathrm{c})$.
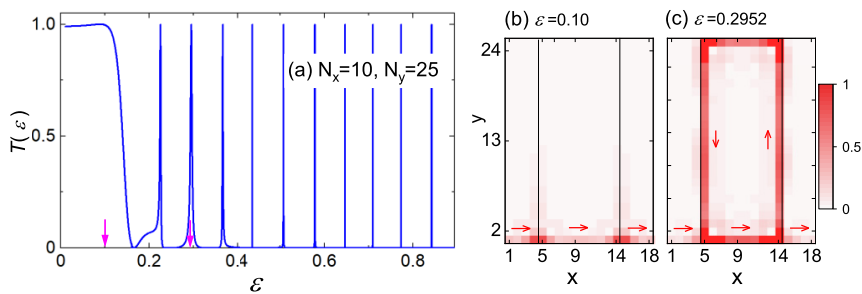

FIG. 4. (a) $T(\varepsilon)$ versus $\varepsilon$ for the double junction depicted in Fig. 1(c) with $N_{x}=10, N_{y}=25, \eta_{L}=\eta_{C}=\eta_{R}=1$, and $\kappa=0.1$. (b) Intensity map of $\sum_{i=s, p}|\psi(x, y, i)|^{2}$, the charge density of the 1D edge state incident from $x=-\infty$ with $\varepsilon=0.1$. (c) The same as panel (b), but at the resonance energy $\varepsilon=0.2952$. The two vertical arrows in panel (a) indicate the $\varepsilon$ values for panels (b) and (c).

\section{B. Junctions with rectangular islands between two semi-infinite ribbons}

Next, we consider the double-interface configuration shown in Fig. 1(c). In the present work, we discuss only the case with $\eta_{L}=\eta_{C}=\eta_{R}=1$ so that the up-spin 1D edge states propagate anticlockwise along the boundaries of all ribbons. The coupling $\kappa$ is assumed to be the same at both interfaces. As in the preceding section, when $\kappa=1$, the system becomes an infinite $1 \mathrm{D}$ crystal so that $T(\varepsilon)=1$ for any $\varepsilon$.

In Fig. 4(a), we show $T(\varepsilon)$ as a function of $\varepsilon$ for a junction in which a single island with $N_{x} \times N_{y}=10 \times 25$ is weakly linked to the two semi-infinite ribbons on both sides with coupling strength $\kappa=0.1$. Compared to the single-interface case discussed above, it is seen that $T(\varepsilon)$ now exhibits a completely different behavior in the upper-energy region. As in Figs. 3(a) and 3(c), for $|\varepsilon|<\varepsilon_{g}^{y} / 2, T(\varepsilon)$ is close to unity since electrons cannot propagate in the positive $y$ direction when $\varepsilon$ is within the energy gap of the 1D interface edge modes. As an example, we show in Fig. 4(b) the squared amplitude of the electron wave function at $\varepsilon=0.1$ located within the transmittance plateau. As is seen, the charge density is mostly localized at the lower edge through the three ribbons.

In striking contrast to Figs. 3(a) and 3(c), $T(\varepsilon)$ for higher energies exhibits sharp peaks instead of decaying oscillatory behavior. These peaks originate from the discrete energy levels of $1 \mathrm{D}$ edge states localized at the boundary of the central island. They become resonances with finite energy widths when coupled to the two semi-infinite ribbons on both sides. Thus, electrons incident from $x=-\infty$ along the lower edge of the left ribbon undergo resonant tunneling through these resonances formed on the island. To demonstrate this, we show in Fig. 4(c) the squared amplitude of the electron wave function at $\varepsilon=0.2962$, which corresponds to the second resonant peak marked by a vertical arrow in Fig. 4(a). As is seen, the charge density exhibits large amplitudes along the four sides of the central island.

The resonant peaks in $T(\varepsilon)$ in Fig. 4(a) can be well described by

$$
T(\varepsilon)=\frac{4 \gamma_{L} \gamma_{R}}{\left(\varepsilon-\varepsilon_{0}\right)^{2}+\left(\gamma_{L}+\gamma_{R}\right)^{2}},
$$

obtained by setting $\hat{\Gamma}_{L}(\varepsilon)=2 \gamma_{L}, \hat{\Gamma}_{R}(\varepsilon)=2 \gamma_{R}$, and $\hat{G}(\varepsilon)=$ $\left[\left(\varepsilon-\varepsilon_{0}\right)+i\left(\gamma_{L}+\gamma_{R}\right)\right]^{-1}$ in Eq. (5), where $\varepsilon_{0}$ gives the center 
of the resonance. Since $\gamma_{L}=\gamma_{R}$ in the present symmetric geometry, $100 \%$ transmission occurs at $\varepsilon=\varepsilon_{0}$. The same result is found to hold for the remaining resonant peaks in Fig. 4(a). We also note that the values of $\gamma_{L}$ are extremely small for some resonant peaks. For example, $\gamma_{L}$ is only $\sim 1.5 \times 10^{-7}$ for the fourth resonant peak at $\varepsilon=0.4371$.

The properties of the double junction discussed above might be potentially useful for applications, as can be illustrated as follows. Let us consider the ballistic conductance of the 1D edge channel, $G=I / V_{b}$, where $I$ is given by Eq. (6) and $V_{b}=\left(\mu_{L}-\mu_{R}\right) / e$ is the bias voltage. If the interval $\left[\mu_{R}, \mu_{L}\right]$ is within $|\varepsilon|<\Delta \varepsilon_{g}^{y} / 2, G$ is close to the conductance quantum $G_{0}=2 e^{2} / h$. On the other hand, if $\left[\mu_{R}, \mu_{L}\right]$ is outside the plateau and contains a single resonance peak, $G \simeq G_{0} \gamma_{L} /\left(e V_{b}\right)$, whereas, if it contains no peaks, $G \simeq 0$. Thus, if one can choose $V_{b}$ such that $\gamma_{L} \ll e V_{b} \ll \Delta \varepsilon_{g}^{y}$, the two energy regions within and outside the energy gap $\Delta \varepsilon_{g}^{y}$ may be used as "current-on" and "current-off" states of electronic devices, respectively.

In order to remove resonant peaks at higher energies in $T(\varepsilon)$, we have further considered "double-island" junctions in which the left island with Chern number $\eta_{C_{1}}$ made out of $N_{x_{1}} \times N_{y}$ lattice sites and the right island with Chern number $\eta_{C_{2}}$ made out of $N_{x_{2}} \times N_{y}$ sites are linked in series at their ends and sandwiched as a whole between two semi-infinite ribbons. The hopping integrals across the two islands are reduced by using the same parameter $\kappa$ as that across the island and semi-infinite ribbon on both sides. If $N_{x_{1}} \neq N_{x_{2}}$, the discrete edge states in the two islands are formed at different energies, resulting in negligibly small hybridizations between them. As a result, the resonant states become mostly localized in either the left or the right island.

To demonstrate this, we show in Fig. 5(a) the local density of states (DOS) of the two islands (summed over two orbitals and all the lattice points in the island) for a junction with $N_{x_{1}}=$ $10, N_{x_{2}}=12, N_{y}=25, \kappa=0.1$, and $\eta_{L}=\eta_{C_{1}}=\eta_{C_{2}}=\eta_{R}=$ 1 . Here, since most of resonant levels have extremely small energy widths, we introduced a finite imaginary energy $\operatorname{Im} \varepsilon=$ $10^{-3}$ to broaden the DOS peaks. As is seen, except for the few lowest peaks, most resonant peaks of the two DOS curves appear at different energies, indicating that they are mainly localized in one of the two islands.

In Fig. 5(b) we show the calculated $T(\varepsilon)$ for the same junction as in Fig. 5(a). Similarly to Fig. 4(a), the transmittance exhibits a plateau with $T(\varepsilon) \simeq 1$ within the energy gap $|\varepsilon|<$ $\Delta \varepsilon_{g}^{y} / 2$ and several resonant peaks for $|\varepsilon|>\Delta \varepsilon_{g}^{y} / 2$. However, in contrast to Fig. 4(a), where $T\left(\varepsilon_{0}\right)=1$ at all resonant peaks, the resonant levels at higher energies in Fig. 5(a) are hardly seen in Fig. 5(b). (To be more accurate, they are not visible with the vertical-axis scale in this figure.) The reason for this behavior can be understood also with the use of Eq. (7). As stated above, in the present case, most of resonant states are localized in either the left or the right island. Let us consider a resonant level centered at $\varepsilon=\varepsilon_{0}$ localized primarily in the left island. This implies that $\gamma_{L} \gg \gamma_{R}$, and thus we have from Eq. (7) $T\left(\varepsilon_{0}\right)=4 \gamma_{L} \gamma_{R} /\left(\gamma_{L}+\gamma_{R}\right)^{2} \sim 4 \gamma_{R} / \gamma_{L} \ll 1$ at the resonant center. The same holds for resonant states mainly localized in the right island.

In contrast, a few resonant states at low energies produce sizable resonant peaks in $T(\varepsilon)$ since their wave functions are
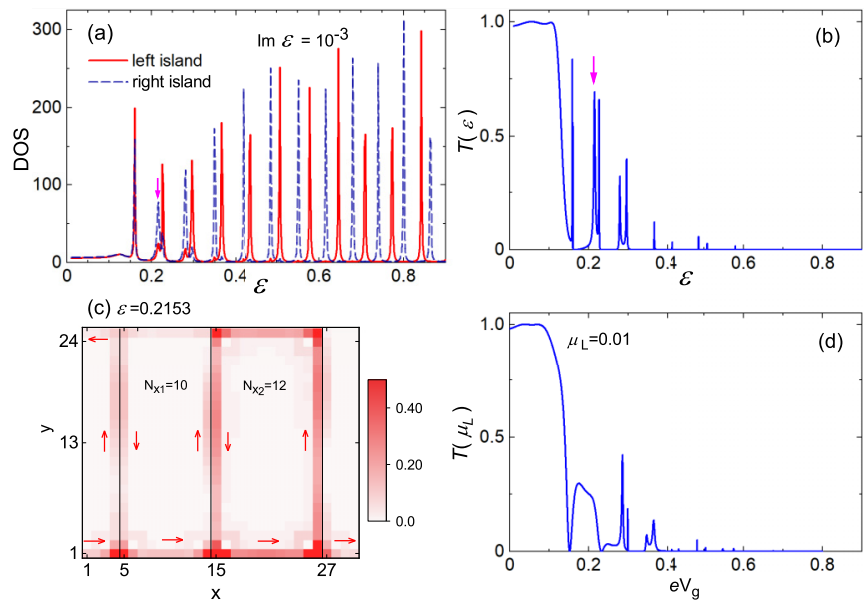

FIG. 5. (a) Local DOS summed over two orbitals and all lattice points within a single island for a "double-island" junction. Solid and dashed lines correspond to the left and right islands with $N_{x_{1}}=10$ and $N_{x_{2}}=12$, respectively. Other parameters are $N_{y}=25, \kappa=0.1$, and $\eta_{L}=\eta_{C_{1}}=\eta_{C_{2}}=\eta_{R}=1$. An imaginary energy $\operatorname{Im} \varepsilon=10^{-3}$ is introduced to broaden DOS peaks. (b) $T(\varepsilon)$ versus $\varepsilon$ for the junction in panel (a). (c) Intensity map of $\sum_{i=s, p}|\psi(x, y, i)|^{2}$, the charge density of the edge state incident from $x=-\infty$ at $\varepsilon=0.2153$ marked by vertical arrows in panels (a) and (b). (d) Transmittance at a constant chemical potential of $\mu_{L}=0.01$ for the same junction as in panel (b) except that the on-site energies of the $s$ and $p$ orbitals within the two islands are lowered by $e V_{g}$ via an external gate voltage.

spread over both island regions. As an example, we show in Fig. 5(c) the squared amplitude of the wave function incident from $x=-\infty$ at $\varepsilon=0.2153$ marked by a vertical arrow in Figs. 5(a) and 5(b). As is seen, the charge density of this resonant state is spread in both islands, although its portion within the left island is considerably smaller than that within the right one. As a consequence, this resonant state emerges as a peak in $T(\varepsilon)$ with $T\left(\varepsilon_{0}\right)=0.695$.

So far we realized the current-on and current-off states in the junction by varying the energy of electrons incident from $x=-\infty$. In realistic experimental setups, this corresponds to varying the chemical potential of the left semi-infinite ribbon, $\mu_{L}$. We now propose another device setup in which the "on" and "off" states of the current through the junction are regulated by applying a gate voltage $V_{g}$ in the island region while fixing $\mu_{L}$ and $\mu_{R}\left(\mu_{L}>\mu_{R}\right)$. To be specific, we assume that the site energies of the $s$ and $p$ orbitals in the two islands are lowered by $e V_{g}$ via an external potential. In this case, when $\kappa=0$, the energy dispersion of the 1D edge mode of the left island, which propagates in the negative $y$ direction, may be written as $\varepsilon \simeq-\alpha\left(k_{y}-\pi\right)-e V_{g}$ with the constant $\alpha$ giving its slope, while that of the semi-infinite left ribbon, which propagates in the positive $y$ direction, is given by $\varepsilon \simeq+\alpha\left(k_{y}-\pi\right)$. When $\kappa>0$, the two modes interact to open an energy gap of size $\Delta \varepsilon_{g}^{y}$ with its center located at $\varepsilon \sim-e V_{g} / 2$. The energy gap at the interface between the right island and the semi-infinite right ribbon is opened in the same energy range. Thus, we expect that a plateau with $T(\varepsilon) \simeq 1$ may emerge within the energy interval $\left|\varepsilon+e V_{g} / 2\right|<\Delta \varepsilon_{g}^{y} / 2$.

To demonstrate this, we plot in Fig. 5(d) the calculated transmittance for the same double-island junction as in 

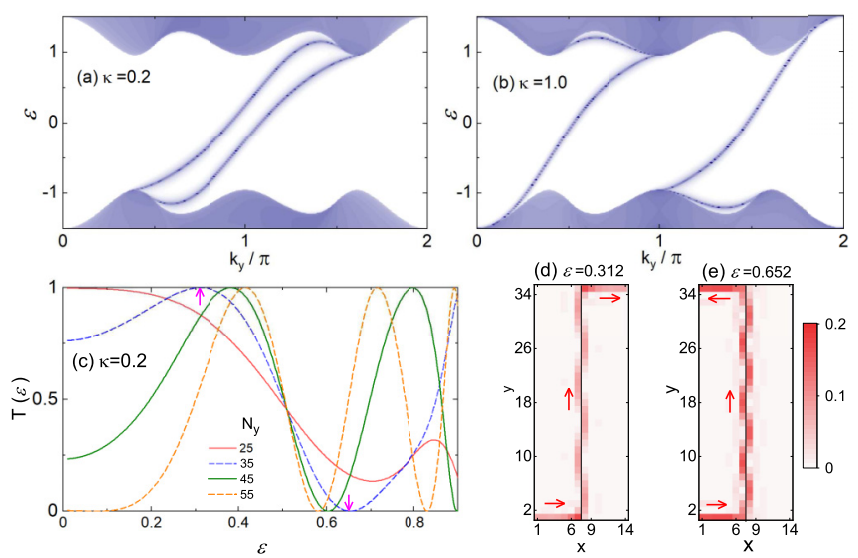

FIG. 6. (a) $\varepsilon$ versus wave number $k_{y}$ of interface edge modes propagating in the positive $y$ direction along the interface for the nanojunction depicted in Fig. 1(b) $\left(\eta_{L}=-\eta_{R}\right)$ with $\kappa=0.2$ and in the limit of $N_{y} \rightarrow+\infty$. (b) The same as panel (a) except for $\kappa=1.0$. (c) Transmittance $T(\varepsilon)$ versus one-electron energy $\varepsilon$ for $\kappa=0.2$. The four curves correspond to $N_{y}=25,35,45$, and 55 . (d) Intensity map of $\sum_{i=s, p}|\psi(x, y, i)|^{2}$, the charge density of the 1D edge state incident from $x=-\infty$ at $\varepsilon=0.312$ marked by the left arrow in panel (c). Red arrows indicate wave propagation directions. The other parameters are $N_{y}=35$ and $\kappa=0.2$. (e) The same as panel (d) except for $\varepsilon=0.652$ indicated by the right arrow in panel (c).

Fig. 5(b) as a function of $e V_{g}$ for a fixed chemical potential, $\mu_{L}=0.01$, which is slightly above the center of the bulk band gap. There is a close similarity between Figs. 5(b) and 5(d). It is therefore feasible that one may make use of the lower $V_{g}$ region with $T\left(\mu_{L}\right) \simeq 1$ as the on state and the higher $V_{g}$ region with $T\left(\mu_{L}\right) \simeq 0$ as the off state.

\section{Junctions between two semi-infinite ribbons with opposite Chern numbers}

As a final application, we consider the single-interface configuration in Fig. 1(b) where the Chern numbers of the valence band for both ribbons are opposite $\left(\eta_{L}=1\right.$ and $\left.\eta_{R}=-1\right)$. Experimentally, it may be difficult to realize such a junction since topological invariants of 2D TIs are bulk properties that cannot be manually changed. The edge states of the two ribbons along the interface, which propagate in the positive $y$ direction and are decoupled from each other at $\kappa=0$, begin to interact with each other with increasing $\kappa$, resulting in two hybridized interface bands crossing the bulk band gap. As an example, we show in Figs. 6(a) and 6(b) the energy dispersion curves of the two bands for $\kappa=0.2$ and 1.0 in the wide ribbon limit $\left(N_{y} \rightarrow+\infty\right)$, respectively. The two bands persist up to $\kappa=1$, since the number of the topological bands crossing the bulk band gap is a bulk property determined by $\left|\eta_{L}+\eta_{R}\right|=2$.

In Fig. 6(c) we plot $T(\varepsilon)$ versus $\varepsilon$ for electrons incident from $x=-\infty$ for a coupling strength of $\kappa=0.2$ and for four $N_{y}$ values. Except for $N_{y}=25$, the $T(\varepsilon)$ curves exhibit oscillating behavior in the whole range between 0 and 1 . Moreover, the interval between two neighboring energy points with $T=0$ and 1 decreases with increasing $N_{y}$.

To achieve a better understanding of this oscillating behavior, we plot in Figs. 6(d) and 6(e) the charge densities of the edge state incident from $x=-\infty$ for $\kappa=0.2$ and $N_{y}=35$ at two energies, $\varepsilon=0.312$ and 0.652 . As marked by vertical arrows in Fig. $6(\mathrm{c}), T(\varepsilon)$ becomes 1 and 0 at these energies, respectively. The charge distributions in both panels exhibit periodic patterns along the interface. One period consists of a maximum on the outermost layer of the right ribbon and the corresponding one of the left ribbon. The wave function of the edge state incident from $x=-\infty$ with energy $\varepsilon=\varepsilon_{0}$ may be expressed by a linear combination of two interface edge modes with wave numbers $k_{y 1}$ and $k_{y 2}$ in the $N_{y}$ wide interface region except near the lower and upper corners. Here, $k_{y 1}$ and $k_{y 2}$ are the intersections of the energy dispersion curves of the two bands shown in Fig. 6(a) and $\varepsilon=\varepsilon_{0}$. Then, one period in the charge density in the $y$ direction is given by $\lambda=2 \pi /\left|\Delta k_{y}\right|$, with $\Delta k_{y}=k_{y 2}-k_{y 1}$. From Fig. 6(a), the $\Delta k_{y}$ values at $\varepsilon=0.312$ and 0.652 are evaluated to be $\simeq 0.55$ and 0.72 , which correspond to $\lambda \simeq 11.4$ and 8.7 , respectively. This indicates that the $N_{y}$ wide interface should contain $N_{y} / \lambda \simeq 3.1$ and 4.0 periodic charge patterns along the $y$ direction, which is in agreement with the charge distributions shown in Figs. 6(d) and $6(\mathrm{e})$. Hence, we conclude that the oscillating behavior of $T(\varepsilon)$ seen in Figs. 6(c) arises from the variation of $\Delta k_{y}$ with $\varepsilon$ and that $T(\varepsilon)$ becomes 0 or 1 when $N_{y} / \lambda$ becomes an integer. We have confirmed that this holds true also for the other $N_{y}$ values in Fig. 6(c).

\section{Discussion}

Before closing we want to discuss to what extent the present results obtained with the BHZ model can be applied to more realistic nanojunctions made out of 2D TIs.

Regarding the transmittance plateau seen in Figs. 3-5, we expect that it is a general property that could occur in any junction in which more than one nanoribbons with the same topological invariant are joined in series. First of all, when two nanoribbons with the same topological invariant are joined together, the topological interface bands crossing the bulk band gap must disappear. However, this does not mean that these bands vanish suddenly when the coupling between the two ribbons is switched on. Instead, the topological surface bands of the left and right ribbons, whose energy dispersion curves intersect each other at some $k_{y}$, start to interact with each other with increasing coupling strength, which may result in the opening of a local energy gap similar to that in Fig. 3(b). Within this energy gap, the edge-state electrons incident from the interior of the left ribbon cannot propagate parallel to the interface, so that all of them are transferred to the right ribbon, crossing the interface. It is likely that this simple mechanism may hold for systems other than the BHZ Hamiltonian. Similarly, when a rectangular island is put between the left and right ribbons and weakly coupled to them, the discrete edge states in isolated islands become sharp resonances. Then, the electrons incident from the left ribbon may undergo resonant tunneling through them independently of the details of the Hamiltonian.

Since the rectangular islands considered in Sec. III B can be regarded as $2 \mathrm{D}$ TI quantum dots, it may be intriguing to ask whether the Coulomb blockade may influence transport properties of the junctions. As seen from Figs. 4(a) and 5 (b), the edge-state electrons exhibit completely different 
transport properties depending on whether their energies are within or outside the local energy gap $\Delta \varepsilon_{g}^{y}$. Outside the gap, the edge-state electrons in the island can circle around the whole perimeter of the island and form bound states when their wave function satisfies the periodic boundary condition. Furthermore, via the coupling to the left and right ribbons, they become resonances, through which edge-state electrons tunnel from the left to the right ribbon. In this case, the Coulomb blockade would influence the electron transport at low temperatures if the energy width of the resonances becomes smaller than the Coulomb repulsion energy between two electrons localized in the island [42]. On the other hand, as seen from Fig. 5(a), the local DOS in the island is nearly constant within the energy gap $(|\varepsilon|<0.13$ for $\kappa=0.1)$, indicating that no resonances are formed. Within the energy gap, the electron wave function as a function of $y$ is localized at the lower boundary of the ribbons because the edge states cannot propagate in the $y$ direction. Since the edge-state electrons at the lower boundary of the island cannot be backscattered at both ends, they cannot form sin-wave-like bound states. This is the reason why no resonant states are formed within the energy gap. In this energy range, the amplitude of electron wave functions is rather uniform from $x=-\infty$ to $+\infty$, exhibiting no enhancement in the island, no matter how small the coupling strength of the island to neighboring ribbons is. Thus, we expect that no Coulomb blockade occurs within the energy gap $\Delta \varepsilon_{g}^{y}$ and the transmission plateau would persist.

As a final remark, we point out that in order for the transmittance oscillation as seen in Fig. 6(c) to occur, it is not necessary that the electronic states in the left ribbon with $\eta_{L}=$ 1 and those in the right ribbon with $\eta_{R}=-1$ be energetically degenerate as assumed in Sec. III C. Instead, it suffices that the left and right ribbons possess the opposite Chern numbers. The point is that the number of the topological bands crossing the bulk band gap is a bulk property determined by $\left|\eta_{L}-\eta_{R}\right|$, so that there must be two interface bands if $\eta_{L}=1$ and $\eta_{R}=-1$. In this case, the wave function of an edge state propagating parallel to the interface is a superposition of these two interface states, and the interference of both components would give rise to an oscillatory behavior in the transmittance as a function of energy.

\section{SUMMARY}

In the present paper, we studied the ballistic electron current through a junction comprising 2D TI ribbons joined in series. In the simplest case, the junction consists of two semi-infinite narrow ribbons having the same topological invariant. When both ribbons are weakly coupled at their ends, their 1D edge states interact with each other, leading to the opening of an energy gap. Within this energy gap, 1D edge states incident from the interior of one of the ribbons are nearly perfectly transmitted across the junction, whereas outside the energy gap, the transmittance exhibits oscillations which decay with increasing energy. We have further considered junctions where a rectangular island of 2D TI is sandwiched between the semi-infinite ribbons. In this case, the low-energy transmission plateau within the local energy gap persists, whereas the decaying oscillations at higher energies are replaced by sharp conduction peaks due to resonant levels formed in the island. We have shown that these resonant peaks can be reduced by inserting a second rectangular island between the semi-infinite ribbons. Based on these numerical results, we proposed to use the low-energy transmittance plateau as a current-on state and the higher energy region with negligibly small conductance as a current-off state in transistorlike electronic devices.

In order to develop such devices that function at room temperatures, one would need 2D TIs with large bulk band gaps. We note that extensive research in this direction is currently under way [12,17-23].

\section{ACKNOWLEDGMENTS}

This work is supported by the Japan Society for the Promotion of Science (JSPS) KAKENHI, Grant No. JP18H01875. One of the authors (H.I.) is grateful to the Alexander von Humboldt Foundation for support during his stay in Jülich.
[1] C. L. Kane and E. J. Mele, $\mathrm{Z}_{2}$ Topological Order and the Quantum Spin Hall Effect, Phys. Rev. Lett. 95, 146802 (2005).

[2] C. L. Kane and E. J. Mele, Quantum Spin Hall Effect in Graphene, Phys. Rev. Lett. 95, 226801 (2005).

[3] M. Z. Hasan and C. L. Kane, Colloquium: Topological insulators, Rev. Mod. Phys. 82, 3045 (2010).

[4] B. A. Bernevig and T. L. Hughes, Topological Insulators and Topological Superconductors (Princeton University, Princeton, NJ, 2013).

[5] A. Bansil, H. Lin, and T. Das, Colloquium: Topological band theory, Rev. Mod. Phys. 88, 021004 (2016).

[6] D. Vanderbilt, Berry Phases in Electronic Structure Theory (Cambridge University, Cambridge, England, 2018).

[7] M. G. Vergniory, L. Elcoro, C. Felser, N. Regnault, B. A. Bernevig, and Z. Wang, A complete catalog of high-quality topological materials, Nature (London) 566, 480 (2019).
[8] D. Culcer, A. C. Keser, Y. Li, and G. Tkachov, Transport in two-dimensional topological materials: Recent developments in experiment and theory, 2D Mater. 7, 022007 (2020).

[9] B. A. Bernevig, T. L. Hughes, and S. C. Zhang, Quantum spin Hall effect and topological phase transition in $\mathrm{HgTe}$ quantum wells, Science 314, 1757 (2006).

[10] M. König, S. Wiedmann, C. Brüne, A. Roth, H. Buhmann, L. W. Molenkamp, X. L. Qi, and S. C. Zhang, Quantum spin Hall insulator state in HgTe quantum wells, Science 318, 766 (2007).

[11] A. Roth, C. Brüne, H. Buhmann, L. W. Molenkamp, J. Maciejko, X.-L. Qi, S.-C. Zhang, Nonlocal transport in the quantum spin Hall state, Science 325, 294 (2009).

[12] T. Olsen, E. Andersen, T. Okugawa, D. Torelli, T. Deilmann, and K. S. Thygesen, Discovering two-dimensional topological insulators from high-throughput computations, Phys. Rev. Mater. 3, 024005 (2019). 
[13] S. Murakami, Quantum Spin Hall Effect and Enhanced Magnetic Response by Spin-Orbit Coupling, Phys. Rev. Lett. 97, 236805 (2006).

[14] M. Wada, S. Murakami, F. Freimuth, and G. Bihlmayer, Localized edge states in two-dimensional topological insulators: Ultrathin Bi films, Phys. Rev. B 83, 121310(R) (2011).

[15] F. Yang, L. Miao, Z. F. Wang, M.-Y. Yao, F. Zhu, Y. R. Song, M.-X. Wang, J.-P. Xu, A. V. Fedorov, Z. Sun et al., Spatial and Energy Distribution of Topological Edge States in Single Bi(111) Bilayer, Phys. Rev. Lett. 109, 016801 (2012).

[16] L. Peng, J.-J. Xian, P. Tang, A. Rubio, S.-C. Zhang, W. Zhang, and Y.-S. Fu, Visualizing topological edge states of single and double bilayer $\mathrm{Bi}$ supported on multibilayer $\mathrm{Bi}(111)$ films, Phys. Rev. B 98, 245108 (2018).

[17] Y. Xu, B. Yan, H.-J. Zhang, J. Wang, G. Xu, P. Tang, W. Duan, and S.-C. Zhang, Large-Gap Quantum Spin Hall Insulators in Tin Films, Phys. Rev. Lett. 111, 136804 (2013).

[18] Z. Ni, E. Minamitani, Y. Ando, and S. Watanabe, Germanene and stanene on two-dimensional substrates: Dirac cone and $Z_{2}$ invariant, Phys. Rev. B 96, 075427 (2017).

[19] D. Di Sante, P. Eck, M. Bauernfeind, M. Will, R. Thomale, J. Schäfer, R. Claessen, and G. Sangiovanni, Towards topological quasifreestanding stanene via substrate engineering, Phys. Rev. B 99, 035145 (2019).

[20] X. Qian, J. Liu, L. Fu, and J. Li, Quantum spin Hall effect in two-dimensional transition metal dichalcogenides, Science 346, 1344 (2014).

[21] Z. Fei, T. Palomaki, S. Wu, W. Zhao, X. Cai, B. Sun, P. Nguyen, J. Finney, X. Xu, and D. H. Cobden, Edge conduction in monolayer $\mathrm{WTe}_{2}$, Nat. Phys. 13, 677 (2017).

[22] S. Tang et al., Quantum spin Hall state in monolayer $1 T^{\prime}-\mathrm{WTe}_{2}$, Nat. Phys. 13, 683 (2017).

[23] A. Lau, R. Ray, D. Varjas, and A. R. Akhmerov, Influence of lattice termination on the edge states of the quantum spin Hall insulator monolayer $1 T^{\prime}-\mathrm{WTe}_{2}$, Phys. Rev. Mater. 3, 054206 (2019).

[24] G. M. Gusev, Z. D. Kvon, O. A. Shegai, N. N. Mikhailov, S. A. Dvoretsky, and J. C. Portal, Transport in disordered twodimensional topological insulators, Phys. Rev. B 84, 121302(R) (2011).

[25] G. M. Gusev, Z. D. Kvon, E. B. Olshanetsky, and N. N. Mikhailov, Mesoscopic transport in two-dimensional topological insulators, Solid State Commun. 302, 113701 (2019).

[26] L. Sheng, D. N. Sheng, C. S. Ting, and F. D. M. Haldane, Nondissipative Spin Hall Effect via Quantized Edge Transport, Phys. Rev. Lett. 95, 136602 (2005).

[27] J. I. Väyrynen, M. Goldstein, and L. I. Glazman, Helical Edge Resistance Introduced by Charge Puddles, Phys. Rev. Lett. 110, 216402 (2013).
[28] G. Kirczenow, Perfect and imperfect conductance quantization and transport resonances of two-dimensional topologicalinsulator quantum dots with normal conducting leads and contacts, Phys. Rev. B 98, 165430 (2018).

[29] S. Tiwari, M. L. Van de Put, B. Sorée, and W. G. Vandenberghe, Carrier transport in two-dimensional topological insulator nanoribbons in the presence of vacancy defects, 2D Mater. 6, 025011 (2019).

[30] L. A. Wray, Topological transistor, Nat. Phys. 8, 705 (2012).

[31] Q. Liu, X. Zhang, L. B. Abdalla, A. Fazzio, and A. Zunger, Switching a normal insulator into a topological insulator via electric field with application to phosphorene, Nano Lett. 15, 1222 (2015).

[32] W. G. Vandenberghe and M. V. Fischetti, Imperfect twodimensional topological insulator field-effect transistors, Nat. Commun. 8, 14184 (2017).

[33] Y. Xu, Y.-R. Chen, J. Wang, J.-F. Liu, and Z. Ma, Quantized Field-Effect Tunneling between Topological Edge or Interface States, Phys. Rev. Lett. 123, 206801 (2019).

[34] Y. Takagaki, Conductance fluctuations induced by bulk state in quasi-one-dimensional strips of topological insulator, Phys. Rev. B 85, 155308 (2012).

[35] Y. Takagaki, Quantum interference by localized scattering waves of gapless helical modes in narrow strips of topological insulators, J. Appl. Phys. 120, 124304 (2016).

[36] L. Fu and C. L. Kane, Topological insulators with inversion symmetry, Phys. Rev. B 76, 045302 (2007).

[37] X. Dang, J. D. Burton, A. Kalitsov, J. P. Velev, and E. Y. Tsymbal, Complex band structure of topologically protected edge states, Phys. Rev. B 90, 155307 (2014).

[38] J. E. Inglesfield, The Embedding Method for Electronic Structure (IOP, Bristol, 2015).

[39] M. Brandbyge, J. L. Mozos, P. Ordejón, J. Taylor, and K. Stokbro, Density-functional method for nonequilibrium electron transport, Phys. Rev. B 65, 165401 (2002).

[40] Y. Xue and M. A. Ratner, Microscopic study of electrical transport through individual molecules with metallic contacts. I. Band lineup, voltage drop, and high-field transport, Phys. Rev. B 68, 115406 (2003).

[41] H. Ishida, A. Liebsch, and D. Wortmann, Topological invariants of band insulators derived from the local-orbital based embedding potential, Phys. Rev. B 96, 125413 (2017).

[42] H. Ishida and A. Liebsch, Coulomb blockade and Kondo effect in the electronic structure of Hubbard molecules connected to metallic leads: A finite-temperature exact-diagonalization study, Phys. Rev. B 86, 205115 (2012). 